\title{
Equipment
}

Mark J. Cleland BMET,

Ba Pham MMATH, Donald R. Miller MD

\section{Influence of arrhythmias on accuracy of non-inva- sive blood pressure monitors}

\author{
Purpose: To compare the accuracy of non-invasive blood pressure (NIBP) monitors in response to common car-
} diac arrhythmias.

Methods: Simulated signals of normal sinus rhythm (NSR), premature ventricular contractions (PVCs), atrial fibrillation (AF) and missed beats (MB) were generated from a Cufflink ${ }^{\text {TM }}$ (Dynatech Nevada) NIBP simulator. Using these signals, the Critkon 1846SX (C/846), Critikon 845xt (C845), Critikon Vital Signs (CVIT), and Hewlett Packard MI008a (HPI 008) were studied at a standard dynamic blood pressure of $120 / 90 / 80 \mathrm{mmHg}$, in order to compare monitor accuracy and signal response times.

Results: The C 845 monitors most closely estimated a simulated SBP of $120 \mathrm{mmHg}$, although SBP was greater during PVCs and AF than NSR(P < 0.05). The Critikon 1846, Critikon Vital Signs, and Hewlett Packard systematically underestimated SBP during these arrhythmias, but variability was modest, as reflected by small coefficients of variation $(<2 \%$ for SBP) with all monitor types. In general, MAP and DBP were less sensitive to the effects of these arrhythmias. Finally, missed beats prolonged signal response times with all four monitor types $(P<0.05)$, whereas PVCs and AF did not alter this parameter.

Conclusions: This study demonstrates the extent to which the accuracy of NIBP monitors is altered by common cardiac arrhythmias. Differences in the electromechanical characteristics of these devices may help to explain the observed similarities and discrepancies.

Objectif : Comparer la précision de différents moniteurs de tension artérielle non effractifs (TANE), lors d'arythmies cardiaques habituelles.

Méthodes : Des signaux simulés de rythme sinusal normal (RSN), d'extrasystoles ventriculaires (EV), de fibrillation

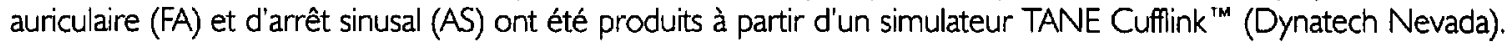
En nous servant de ces signaux, nous avons étudié le Critikon 18465X (Cl 846), le Critikon 845xt (C845), le Critikon Vital Signs (CVIT), et le Hewlett Packard MI008a (HPI008) selon une tension artérielle dynamique standard de 120/90/80 $\mathrm{mmH}$, dans le but de comparer la précision des moniteurs et les temps de réponse.

Résultats : Le moniteur C845 a fourni l'estimation la plus juste d'un tension artérielle systolique (TAS) simulée de $120 \mathrm{mmHg}$, bien que la TAS a été meilleure pendant l'EV et la FA que pendant le RSN $(P<0,05)$. Le Critikon I846, le Critikon Vital Signs et le Hewlett Packard ont systématiquement sous-estimé la TAS pendant ces arythmies, mais la variabilité a été peu importante, comme l'indiquent les faibles coefficients de variation ( $<2 \%$ pour la TAS) pour tous les types de moniteurs. En général, la tension artérielle moyenne (TAM) et la tension artérielle diastolique (TAD) ont été moins sensibles aux effets de ces arythmies. Finalement, l'arrêt sinusal a prolongé le temps de réponse avec les quatre types de moniteurs $(P<0,05)$, alors que les $E V$ et la $F A$ riont pas modifié ce paramètre.

Conclusion : Cette étude montre jusqu'où les arythmies cardiaques courantes modifient la précision des moniteurs de pression artérielle non effractifs. Les différentes caractéristiques électromécaniques des appareils peuvent contribuer à expliquer les similarités et les écarts observés.

\footnotetext{
From the Departments of Biomedical Engineering, Internal Medicine, and Anaesthesia, Ottawa General Hospital and the University of Ottawa, Ottawa, Ontario. Presented, in part, at the Canadian Anaesthetists' Society Annual Meeting, Ottawa, Ontario; June 1995. Address correspondence to: Dr. Donald R. Miller, Department of Anaesthesia, Ottawa General Hospital, 501 Smyth Road, Ottawa, Ontario KlH 8L6 Canada. Phone: 613-737-8187; Fax: 613-737-8189; E-mail: dmiller@ogh.on.ca. Accepted for publication April 22, 1998
} 
$\mathrm{E}$ VALUATION of automated oscillometric non-invasive blood pressure (NIBP) monitors has traditionally involved a simultaneous comparison with either manual mercury sphygmomanometers (auscultatory method), or direct intra-arterial pressure measurements. ${ }^{1-7}$ A limitation inherent to these types of comparisons is that they systematically evaluate two or more distinct pressure entities. With the recent development of NIBP simulators, static and dynamic pressure simulations are now possible. ${ }^{8}$ Test equipment capable of performing dynamic blood pressure simulations provides a benchmark against which NIBP monitors can be tested in order to determine both accuracy and reproducibility. In addition, signal response times can also be measured. This technology offers advantages over manual methods by eliminating observer subjectivity, and by offering an unprecedented degree of reproducibility between subsequent readings.

Software has now been developed for NIBP simulators which incorporates algorithms for arrhythmia simulation. Common arrhythmias, including premature ventricular contractions (PVC), atrial fibrillation (AF) and missed beats $(\mathrm{MB})$ can be introduced into the NIBP monitor's blood pressure determination.

While it has been suggested that these, and possibly other cardiac arrhythmias may prolong the response times and accuracy of NIBP monitors, ${ }^{9}$ no systematic evaluation of these devices has been conducted. Therefore, a prospective evaluation of NIBP monitors at one tertiary care hospital was undertaken, in order to evaluate the effects of PVCs, AF and MB on both accuracy and monitor response times.

\section{Methods}

All non-invasive blood pressure monitors in use throughout the operating rooms and critical care areas of the Ottawa General Hospital, a 400-bed adult tertiary care hospital, were tested. A Cufflink ${ }^{\mathrm{TM}}$ NIBP simulator (Dynatech, Nevada) provided the dynamic pressure simulations as well as a benchmark with which all monitors could be compared. This machine, which was calibrated prior to the study, has a reported accuracy of $\pm 1 \%$ over simulated BP ranges of $60 / 50 / 30$ to $250 / 200 / 180 \mathrm{mmHg}$ (corresponding to systolic, mean, and diastolic blood pressures, respectively). ${ }^{a}$ Blood pressure readings and response times for each simulation were obtained via the Cufflink ${ }^{\mathrm{TM}}$ device. Measurements included: simulated pressure $(120 / 90 / 80 \mathrm{mmHg})$, inflate time (sec) inflation rate $\left(\mathrm{mmHg} \cdot \mathrm{sec}^{-1}\right)$, deflate time $(\mathrm{sec})$ and deflation rate $\left(\mathrm{mmHg} \cdot \mathrm{sec}^{-1}\right.$ ), as well as acquisition time.

All tests were performed by two experienced technicians in the institutional Biomedical Engineering
Department. The test setup was established by placing the simulator in-line to the Cufflink ${ }^{\mathrm{TM}}$, via a " $\mathrm{t}$ " connector attached to the monitor's hose and cuff assembly. The monitor's cuff was then secured around a mock plastic arm with a diameter of $35 \mathrm{~cm}$ (approximate size of an average adult arm). During the arrhythmia simulation, the NIBP monitor automatically interpreted the simulated pressure pulses and responses once each blood pressure signal was generated. The measured responses from the monitors' blood pressure readings were transcribed onto the Cufflink's ${ }^{\mathrm{TM}}$ summary report.

A comparison of signal response times (from trigger to measurement reading) and accuracy of four models of non-invasive blood pressure (NIBP) monitors was undertaken. The following four NIBP monitors were tested: Critikon 1846SX (C1846), Critikon 845xt (C845), Critikon Vital Signs (CVIT), and Hewlett Packard M1008a (HP1008). Each monitor was subjected to three blood pressure assessments at $120 / 90 / 80 \mathrm{mmHg}$. This reflected systolic, mean and diastolic blood pressures, for each of the following cardiac rhythms: 1) NSR, 2) PVC, 3) AF, and 4) MB. Arrhythmia morphology templates were used which had the following characteristics:

i) Premature ventricular contractions were randomly generated at a rate of approximately $6 \cdot \mathrm{min}^{-1}$. The PVC amplitude was reduced to $85 \%$ of a normal pulse, with a $15 \%$ reduction in pulse width. During the subsequent pulse, amplitude was restored while maintaining the shortened duration, while the next beat was generated with restoration of both amplitude and pulse.

ii) Atrial fibrillation was generated by altering both pulse width and amplitude by 85 to $115 \%$ of a normal beat, while the next beat was delayed the reciprocal amount with a corresponding change in gain. The ventricular response rate during simulated AF could be calculated by multiplying the new altered beat by a factor of 80 .

iii) Missed beats were randomly generated by delaying the normal pulse 8-12 sec before returning to the NSR mode.

\section{Statistical analysis}

The accuracy of the monitors was based upon three measures: (1) the measured blood pressure; (2) the bias in the reading (i.e. the difference between the observed reading and the simulated standard value),

\footnotetext{
${ }^{\mathrm{a}}$ Cufflink ${ }^{\mathrm{TM}}$ Non-Invasive Blood Pressure Analyzer: Operating and Service Manual; Dynatech Nevada Inc. 1996.
} 
and (3) the coefficient of variation (CV or signal-versus-noise ratio) ${ }^{10}$ derived from three replicates for each monitor at each arrhythmia. The signal-versusnoise ratio captured the variation in response that did not depend upon the mean. The following variables were considered in the analysis: SBP, MAP, DBP and signal response time. A desired outcome was considered to be a response mean level close to the 'target', with a small coefficient of variation.

The major factors which might have influenced response outcomes included monitor type, rhythm and their interaction. Factor effects were assessed in an analysis of variance (ANOVA) model in which the number of monitors per monitor type was considered a small random sample from a very large batch for each model (i.e. a mixed-effect model). ${ }^{11}$ Estimated mean responses and their $95 \%$ confidence intervals for the two major factors were derived from the model. Arrhythmias were compared to normal sinus rhythm by checking for overlapping of their respective $95 \%$ confidence intervals.

For each variable, mean values, $95 \%$ confidence intervals, bias, and coefficients of variation are presented in tabular format. In addition, box and whisker plots are provided to give a visual presentation of mean values $\pm 95 \%$ confidence intervals (the 'boxes'), in addition to the ranges (the 'whiskers'). Extreme observations were defined as data points occurring beyond $\pm 2 \mathrm{SD}$ of mean values. Statistical significance was assumed when $P<0.05$, without adjustment for multiple comparisons.

\section{Results}

Sixty one monitors were evaluated: C1846 $(n=21)$; C845 ( $n=11)$; CVIT $(n=4)$; and HP1008 $(n=25)$. The smaller number of CVIT monitors resulted in the $95 \%$ confidence intervals subsuming the actual range of values during most of the simulated arrhythmias. This occurred since the related standard error used to derive the confidence intervals was estimated from the model, including all monitors with substantial variation.

The influence of the simulated arrhythmias on the accuracy of SBP measurements is displayed in Figure 1 and Table I. Overall, the C845 estimated most closely the simulated value of $120 \mathrm{mmHg}$ for all four cardiac rhythms, despite the fact that recorded SBP readings increased during PVCs $(P<0.05)$ and AF $(P<0.05)$. In contrast, the other three NIBP monitors demonstrated a consistent trend to underestimation of SBP during simulated PVCs, AF, and MB (compared with NSR). The coefficient of variation was $2 \%$ for the Critikon 845 and less than $1 \%$ for the Hewlett Packard M1008 and the Critikon 1846. All five extreme observations belonged to the Critikon 845 .
Results of the mean arterial pressure (MAP) analysis are displayed in Figure 2 and Table II. While overall mean values were within $\pm 8 \%$ of the simulated value 90 $\mathrm{mmHg}$ for each of the four cardiac rhythms, atrial fibrillation (AF) resulted in an overestimation of MAP with the Critikon 845 ( $P<0.05$ compared with NSR). Systematic error was also observed with other monitor types (e.g. + $3.8 \mathrm{mmHg}$ with the Critikon 845; and $3.3 \mathrm{mmHg}$ with the Hewlett Packard M1008) under the different rhythms. However, coefficients of variation were small, ranging from $0.9 \%$ with the Critikon 1846 to $3.1 \%$ with the Critikon 845 . Of the nine extreme observations, eight were from the Critikon 845 and one was from the Critikon 1846.

Figure 3 and Table III summarize the diastolic blood pressure (DBP) data. Errors ranged from $+0.7 \mathrm{mmHg}$ for the Critikon 845 to $-9.5 \mathrm{mmHg}$ for the Hewlett Packard Ml008, with a systematic error across all rhythm levels in each monitor. Variation due to monitors was estimated to be $12 \%$ of the repeated observations variation (ie. within monitor and rhythm). Coefficients of variation ranged from $1.3 \%$ for the Critikon 1846, to $2.5 \%$ for the Hewlett Packard. There were 10 extreme observations with $\mathrm{HP}$ M1008 and three with the Critikon 845. Overall, the HP measures were furthest from the $80 \mathrm{mmHg}$ target for all simulated arrhythmias.

Figure 4 and Table IV summarize results from the response-time analysis. For all four monitor types, $\mathrm{MB}$ resulted in an increase in signal response time com-



FIGURE 1 Box and whisker plots of systolic blood pressure (SBP) in response to a simulated SBP of $120 \mathrm{mmHg}$ during normal sinus rhythm, premature ventricular contractions, atrial fibrillation and missed beats. Plots display mean values, $\pm 95 \%$ confidence intervals around the means (the 'boxes'), in addition to the ranges ('whiskers'). C1846 = Critikon $1846, \mathrm{C} 845=$ Critikon 845, CVIT = Critikon Vital Signs, HP1008 = Hewlett Packard M1008a. Note that the 95\% CI of CVIT mean values subsumed their actual ranges for simulated SBP, MAP and DBP. 
TABLE I Systolic Blood Pressure (mmHg): Mean (95\% Confidence Interval ${ }^{l}$ ) [Bias ${ }^{2}$ ]/ Coefficient of variation ${ }^{3}$ (95\% C.I.)

\begin{tabular}{lllll}
\hline Monitor/Rhythm & NSR & PVC & $A F$ & $M B$ \\
\hline $\begin{array}{l}\text { Critikon } 1846 \mathrm{SX} \\
\left(\mathrm{n}^{4}=21\right)\end{array}$ & $113.6(112.9,114.3)[-6.4]$ & $114.6(113.9,115.3)[-5.4]$ & $114.6(113.9,115.3)[-5.4]$ & $114.5(113.8,115.2)[-5.5]$ \\
& $0.9(0.7,1.2)$ & $0.9(0.7,1.2)$ & $0.8(0.6,1.0)$ & $0.8(0.6,1.0)$ \\
$\begin{array}{l}\text { Critikon 845 XT } \\
(\mathrm{n}=11)\end{array}$ & $120.2(119.2,121.2)[0.2]$ & $122.9(121.8,123.9)[2.9]$ & $123.3(122.3,124.4)[2.3]$ & $122.1(121.0,123.1)[2.1]$ \\
& $2.2(1.6,3.1)$ & $1.9(1.3,2.6)$ & $2.1(1.5,2.9)$ & $1.7(1.2,2.3)$ \\
$\begin{array}{l}\text { Critikon Vital Signs } \\
(\mathrm{n}=4)\end{array}$ & $114.8(110.3,119.2)[-5.2]$ & $113.8(112.1,115.5)[-6.2]$ & $113.9(112.2,115.6)[-6.1]$ & $114.0(112.3,115.7)[-6.0]$ \\
& $\mathrm{n} / \mathrm{a}^{5}$ & $\mathrm{n} / \mathrm{a}$ & $\mathrm{n} / \mathrm{a}$ & \\
HP-M1008A & $114.1(113.4,114.9)[-5.9]$ & $114.7(114.0,115.4)[-5.3]$ & $115.0(114.4,115.7)[-5.0]$ & $115.4(114.7,116.1)[-4.6]$ \\
$(\mathrm{n}=25)$ & $0.8(0.6,1.1)$ & $1.2(0.9,1.5)$ & $0.8(0.7,1.0)$ & $1.2(1.0,1.5)$ \\
\hline
\end{tabular}

1. Estimated mean and its $95 \%$ confidence interval from an analysis of variance model containing two factors: monitor type, rhythm and their interaction. The model results were as follows: monitor type $(P<0.001)$, rhythm $(P<0.001)$ and their interaction $(P=0.12)$. Variation due to monitors (different number of monitors according to monitor type) was estimated to be $31.9 \%$ of variation due to repeated observations per monitor (i.e. triplicates). An underlined response mean was different from the corresponding SNR response at a $5 \%$ significance level. 2. Bias is defined as the difference between an observed SBP with its simulated value of $120 \mathrm{mmHg}$.

3. Coefficient of variation is the ratio between a standard deviation and the corresponding mean, expressed in percentage. Estimated mean and its $95 \%$ C.I. were derived from an analysis of variance model containing the same factors as indicated in 1 . The model response was a monotone function of the coefficient of variation. The model results were as follows: monitor type $(P<0.001)$, rhythm $(P=0.14)$ and their interaction $(P=0.10)$.

4. Number of monitors used in the experiment with available data.

5. " $\mathrm{n} / \mathrm{a}$ " stands for not available due to insufficient number of repeated observations per monitor.

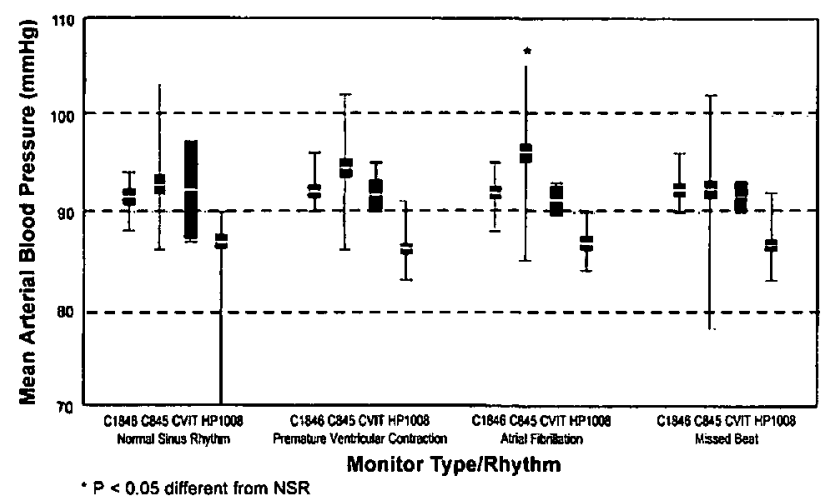

FIGURE 2 Mean arterial pressure (MAP) in response to a simulated MAP of $90 \mathrm{mmHg}$ during normal sinus rhythm, premature ventricular contractions, atrial fibrillation and missed beats. The lower range for HP1008 during NSR was $66 \mathrm{mmHg}$. For a list of abbreviations, refer to the legend of Figure 1 .

pared with NSR $(P<0.05)$. With the Critikon 1846, response times ranged from $18.8 \mathrm{sec}$ during NSR to $26.3 \mathrm{sec}$ with MB. The CVIT monitors had the second fastest response times, requiring $21.4 \mathrm{sec}$ with NSR and 28.6 during MB. Similarly, HP ranged from $23.7 \mathrm{sec}$ during NSR to $32.0 \mathrm{sec}$ with $\mathrm{MB}$; and the Critikon 845 ranged from $32.9 \mathrm{sec}$ during $\mathrm{AF}$ to 41.5 sec with MB. Variations in response times due to monitor type was $47 \%$ of that due to repeated observations for each rhythm and each monitor. Hence, within each monitor type, the acquisition time varied much

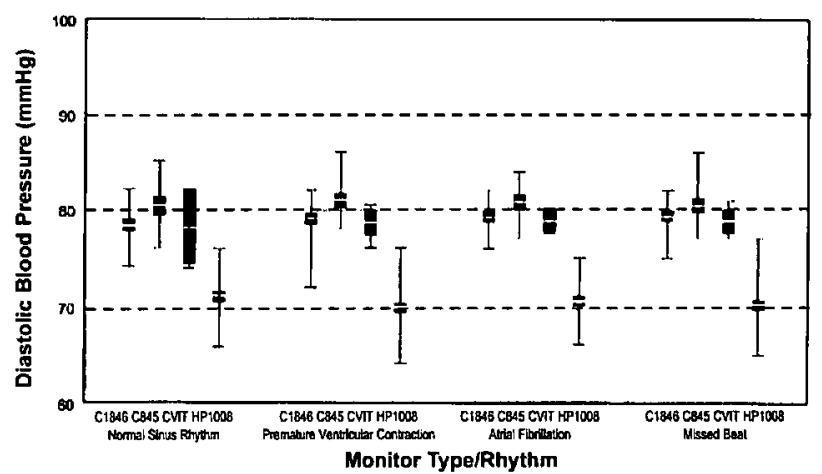

FIGURE 3 Diastolic blood pressure (DBP) in response to a simulated DBP of $80 \mathrm{mmHg}$ during normal sinus rhythm, premature ventricular contractions, atrial fibrillation and missed beats. For a list of abbreviations, refer to the legend of Figure 1 .

more than did MAP-90 from different monitors. Coefficients of variation ranged from a maximum of $8.1 \%$ for both the Critikon 1846 and 845 to a minimum of $5.3 \%$ for the HP. Both the Critikon 1846 and Vital Signs monitor contributed one extreme observation to the response time data.

\section{Discussion}

This study demonstrates the magnitude by which the accuracy of NIBP monitors may be altered by common cardiac arrhythmias (atrial fibrillation, premature 
TABLE II Mean Arterial Pressure (MAP-90) Response: Mean (95\% Confidence Interval ${ }^{1}$ ) $\left[\right.$ Bias $\left.^{2}\right] /$ Coefficient of variation $^{3}$ (95\% C.I.)

\begin{tabular}{llllll}
\hline Monitor/Rhythm & $n^{4}$ & NSR & PVC & $A F$ & $M B$ \\
\hline Critikon 1846 SX & 21 & $91.6(90.9,92.3)[1.6]$ & $92.1(91.4,92.8)[2.1]$ & $92.0(91.3,92.6)[2.0]$ & $92.3(91.6,93.0)[2.3]$ \\
& & $0.9(0.7,1.2)$ & $0.8(0.7,1.0)$ & $0.9(0.7,1.1)$ & $0.9(0.7,1.1)$ \\
Critikon 845 XT & 11 & $92.7(91.8,93.7)[2.7]$ & $94.4(93.5,95.4)[4.4]$ & $96.1(95.1,97.0)[6.1]$ & $92.4(91.4,93.3)[2.4]$ \\
& & $2.7(2.0,3.8)$ & $3.8(2.8,5.3)$ & $4.1(3.0,5.5)$ & $2.7(2.0,3.8)$ \\
Critikon Vital Signs & 4 & $92.2(87.2,97.2)[2.2]$ & $91.8(90.2,93.3)[1.8]$ & $91.2(89.6,92.7)[1.2]$ & $91.6(90.0,93.2)[1.6]$ \\
& & $n / a^{5}$ & $n / a$ & $n / a$ \\
HP-M1008A & 25 & $86.9(86.2,87.6)[-3.1]$ & $86.2(85.6,86.8)[-3.8]$ & $86.8(86.2,87.5)[-3.1]$ & $86.6(86.0,87.2)[-3.4]$ \\
& & $1.6(1.2,2.0)$ & $1.3(1.0,1.6)$ & $1.2(1.0,1.5)$ & $1.8(1.5,2.3)$ \\
\hline
\end{tabular}

1. Estimated mean and its $95 \%$ confidence interval from an analysis of variance model containing two factors: monitor type, rhyclum and their interaction. Both monitor type, rhythm and their interaction were significant $(P<0.001)$. Variation due to monitors (different number of monitors according to monitor type) was estimated to be $8.2 \%$ of variation due to repeated observations per monitor (i.e. triplicates). An underlined response mean was different from the corresponding SNR response at a $5 \%$ significance level.

2. Bias is defined as the difference between an observed MAP with its simulated value of 90 .

3. Coefficient of variation is the ratio between a standard deviation and the corresponding mcan, expressed in percentage. Estimated mean and its $95 \%$ C.I. were derived from an analysis of variance model containing the same factors as indicated in 1 . The model response was a monotone function of the coefficient of variation. The model results were as follows: monitor type $(P<0.001)$, rhythm $(P=0.76)$ and their interaction $(P=0.02)$.

4. Number of monitors used in the experiment with available data.

5 . " $\mathrm{n} / \mathrm{a}$ " stands for not available due to insufficient number of repeated observations per monitor.

TABLE III Diastolic Blood Pressure (mmHg): Mean (95\% Confidence Interval ${ }^{l}$ ) $\left[\right.$ Bias $\left.^{2}\right] /$ Coefficient of variation ${ }^{3}$ (95\% C.I.)

\begin{tabular}{lllll}
\hline Monitor/Rhythm & NSR & PVC & $A F$ & $M B$ \\
\hline $\begin{array}{l}\text { Critikon 1846 SX } \\
\left(\mathrm{n}^{4}=21\right)\end{array}$ & $78.3(77.7,78.9)[-1.7]$ & $79.0(78.4,79.6)[-1.0]$ & $79.2(78.7,79.8)[-0.7]$ & $79.3(78.8,79.9)[-0.7]$ \\
& $1.9(1.5,2.4)$ & $1.4(1.1,1.8)$ & $1.2(0.9,1.6)$ & $1.1(0.9,1.5)$ \\
Critikon 845 XT & $80.4(79.7,81.2)[0.4]$ & $81.0(80.2,81.7)[1.0]$ & $80.8(80.0,81.6)[0.8]$ & $80.4(79.7,81.2)[0.4]$ \\
$(\mathrm{n}=11)$ & $1.7(1.3,2.4)$ & $1.8(1.3,2.5)$ & $1.4(1.0,2.0)$ & $1.8(1.3,2.5)$ \\
Critikon Vital Signs & $78.1(74.3,82.0)[-1.9]$ & $78.6(77.3,79.9)[-1.4]$ & $78.8(77.6,80.1)[1.2]$ & $78.8(77.5,80.0)[1.2]$ \\
$(\mathrm{n}=4)$ & $\mathrm{n} / \mathrm{a}^{5}$ & $\mathrm{n} / \mathrm{a}$ & $\mathrm{n} / \mathrm{a}$ & $\mathrm{n} / \mathrm{a}$ \\
& & & 70.9 & $70.2(69.7,70.7)[9.8]$ \\
HP-M1008A & $71.0(70.4,71.5)[9.0]$ & $69.9(69.4,70.4)[11.1]$ & $70.6(70.1,71.1)[9.4]$ & $7.1(2.5,3.8)$ \\
\hline$(\mathrm{n}=25)$ & $2.5(1.9,3.2)$ & $2.6(2.1,3.3)$ & $2.1(1.7,2.7)$ & 3.1 \\
\hline
\end{tabular}

1. Estimated mean and its $95 \%$ confidence interval from an analysis of variance model containing two factors: monitor type, rhythm and their interaction. The model results were as follows: monitor type $(P<0.001)$, rhythm $(P=0.79)$ and their interaction $(P=0.25)$.

Variation due to monitors (different number of monitors according to monitor type) was estimated to be $12.0 \%$ of variation due to repeatcd obscrvations per monitor (i.e. triplicates).

2. Bias is defined as the difference between an observed DBP with its simulated value of $80 \mathrm{mmHg}$.

3. Coefficient of variation is the ratio between a standard deviation and the corresponding mean, expressed in percentage. Estimated mean and its $95 \%$ C.I. were derived from an analysis of variance model containing the same factors as indicated in 1 . The model response was a monotone function of the coefficient of variation. The model results were as follows: monitor type $(P<0.001)$, rhythm $(P=0.46)$ and thcir interaction $(P=0.37)$.

4. Number of monitors used in the experiment with available data.

5. $\mathrm{n} / \mathrm{a}$ " stands for not available due to insufficient number of repeated observations per monitor.

ventricular contractions and missed beats) at normal blood pressure $(120 / 90 / 80 \mathrm{mmHg})$. The $\mathrm{C} 845$ monitors most closely estimated a simulated SBP of $120 \mathrm{mmHg}$, although SBP was greater during PVCs and AF compared to NSR values. The Critikon 1846, Critikon Vital Signs, and Hewlett Packard systemati- cally underestimated SBP during these arrhythmias, but variability was modest, as reflected by small coefficients of variation (<2\% for SBP) with all monitor types. In general, MAP and DBP were less sensitive to the effects of these arrhythmias. Finally, missed beats prolonged signal response times with all four monitor 
TABLE IV Response Time (sec): Mean (95\% Confidence Interval ${ }^{1}$ ) / Coefficient of variation ${ }^{2}$ ( $95 \%$ C.I.)

\begin{tabular}{lcllll}
\hline Monitor/Rhythm & $\mathrm{n}^{3}$ & NSR & PVC & AF & MB \\
Critikon 1846 SX & 21 & $18.8(17.3,20.3)$ & $19.9(18.4,21.4)$ & $19.3(17.9,20.8)$ & $26.3(24.8,27.8)$ \\
& & $11.5(7.7,17.0)$ & $7.5(5.2,11.0)$ & $5.2(3.6,7.6)$ & $7.6(5.2,11.1)$ \\
Critikon 845 XT & 9 & $35.8(33.5,38.0)$ & $33.0(30.7,35.2)$ & $32.9(30.7,35.2)$ & $41.5(39.3,43.8)$ \\
& & $11.2(6.3,19.9)$ & $4.5(2.5,7.9)$ & $9.5(5.3,16.9)$ & $9.9(5.5,17.5)$ \\
Critikon Vital Signs & 8 & $21.4(19.0,23.8)$ & $23.1(20.7,25.5)$ & $22.5(20.1,24.9)$ & $28.6(26.2,31.0)$ \\
& & $n / a^{4}$ & $n / a$ & $n / a$ & $n / a$ \\
HP-M1008A & 13 & $23.7(21.9,25.6)$ & $25.5(23.6,27.3)$ & $25.4(23.5,27.2)$ & $32.0(30.2,33.9)$ \\
& & $12.4(7.7,20.1)$ & $3.6(2.2,5.8)$ & $2.1(1.3,3.4)$ & $6.8(4.2,11.0)$ \\
\hline
\end{tabular}

1. Estimated mean and its $95 \%$ confidence interval from an analysis of variance model containing two factors: monitor type, rhythm and their interaction. Both monitor type, rhythm and their interaction were significant $(P<0.001)$. Variation due to monitors (different number of monitors according to monitor type) was estimated to be $47 \%$ of variation due to repeated observations per monitor (i.e. triplicates). An underlined response mean was different from the corresponding SNR response at a $5 \%$ significance level.

2. Coefficient of variation is the ratio between a standard deviation and the corresponding mean, expressed in percentage. Estimated mean and its $95 \%$ C.I. were derived from an analysis of variance model containing the same factors as indicated in 1 . The model response An underlined response mean was different from the corresponding SNR response at a $5 \%$ significance level. The model results were as follows: monitor type $(P<0.001)$, rhythm $(P<0.001)$ and their interaction $(P=0.25)$.

3. Number of monitors used in the experiment with available data.

4. " $\mathrm{n} / \mathrm{a}$ " stands for not available due to insufficient number of repeated observations per monitor.

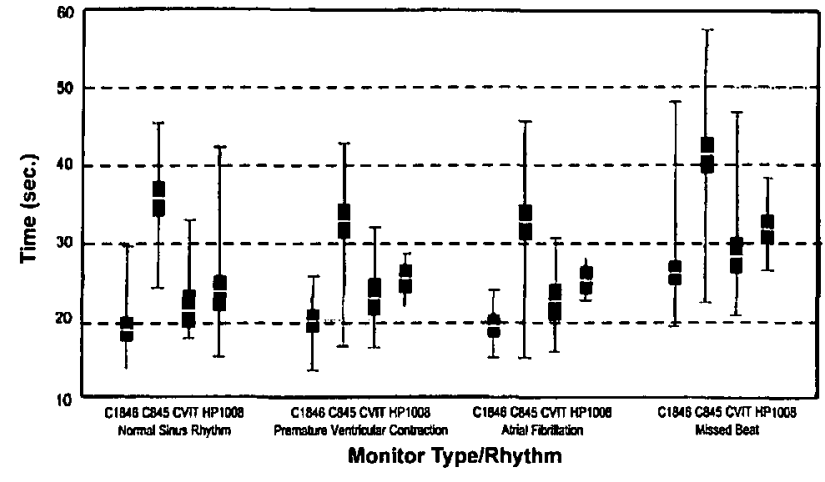

FIGURE 4 Signal response times during normal sinus rhythm premature ventricular contractions, atrial fibrillation and missed beats. For a list of abbreviations, refer to the legend of Figure 1 .

types $(P<0.05)$, whereas PVCs and AF did not alter this parameter.

An explanation of the different accuracies and response times of the four monitors must take into consideration electromechanical characteristics of these devices. Both the NIBP simulator and the test monitors operate using an oscillometric technique. ${ }^{12}$ With this technique, a pneumatic cuff is applied around a limb, and inflated to a pressure above that at which arterial blood flow is occluded. Cuff pressure is then gradually lowered at a rate governed by the NIBP monitor. As cuff pressure decreases, blood flow resumes through the artery, creating low amplitude pressure pulses. While cuff pressure continues to decline, the pressure pulses gradually increase in amplitude. The point at which these pressure pulses reach their maximum amplitude defines the mean arterial pressure. However, the measured pressure pulses represent only 1-3\% of the generated cuff pressure. Accordingly, NIBP monitors may vary in the accuracy with which they subtract the large static cuff pressure from the total pressure, in order to detect the relatively small pressure pulses. Patient motion and respiration are common artefacts which the monitors are designed to reject. Typical artefacts are also eliminated with the NIBP simulator, so that artefacts should not have been an important limitation in this study.

We observed that arrhythmias were associated with longer signal response times with the NIBP monitors which provided the greatest overall level of accuracy; e.g. SBP for C845. As accuracy is closely related to step deflation size, an increased number of steps results, and therefore a longer measurement time would be predicted. This observation did not hold true however, with DBP, which appeared to be insensitive to arrhythmias. One possible explanation is that the fluctuation in rhythms might only have been captured by the most sensitive monitors. Another possibility is that the simulator produces less diastolic pressure variability in individual pulses when simulating arrhythmias.

The Critikon 1846SX NIBP monitor was found to have the most rapid response times. This finding can most readily be explained by the fact that the volume of internal cavity ( 0.8 liters) remains pressurized continuously while the monitor is turned on. The pump reservoir empties into the cuff immediately upon triggering the "start" button. The sole function of the 1846SX pump is to maintain the reservoir's pressure, in contrast to other monitors which rely upon the pump to inflate the cuff. 
Other factors which may influence signal determination include the monitor algorithm's ability to detect the pulsatile signals, and the rate at which the monitor "steps down" the cuff pressure per signal reading. Clinically, acquisition and accuracy can be optimized by proper cuff size selection, positioning of the cuff, ensuring that all hose connections are secure, and by minimizing artefact, motion or electrical interference.

\section{Study limitations}

One limitation of this study is that the data provide an estimate of the $95 \% \mathrm{CI}$ for the mean of each model, rather than the $95 \%$ CI for single readings. Finally, acknowledgment is made of the fact that these data cannot be extrapolated to a range of different systemic blood pressure values. Instead, we chose to study accuracy of NIBP monitors at a standardized value $(120 / 90 / 80 \mathrm{mmHg})$ which is clinically relevant. To have performed the analysis over a range of $B P$ values would have detracted from the primary purpose of the study, in addition to adding unnecessarily to the complexity of the statistical analysis.

\section{Conclusions}

This study demonstrates the extent to which the accuracy of NIBP monitors is altered by common cardiac arrhythmias, where the arrhythmia does not change the patient's haemodynamic state. While the Critikon $845 \mathrm{XT}$ provides the most accurate SBP determinations, these monitors over-estimate SBP in response to PVCs and AF. In contrast, the Critikon 1846, Critikon Vital Signs, and Hewlett Packard monitors systematically underestimate SBP during these arrhythmias. However, variability is modest, as reflected by small coefficients of variation ( $<2 \%$ for SBP) with all monitor types. Furthermore, MAP and DBP are less sensitive to the effects of arrhythmias. Finally, missed beats prolong signal response times with all four monitor types, whereas PVC's and AF do not alter this parameter. Differences in the electromechanical characteristics of these devices may help to explain the observed similarities and discrepancies.

\section{References:}

1 Ling J, Ohara Y, Orime $\Upsilon$, Noon GP, Takatani S. Clinical evaluation of the oscillometric blood pressure monitor in adults and children based on the 1992 AAMI SP-10 standards. J Clin Monit 1995; 11 : 123-30.

2 Young CC, Mark JB, White W, DeBree A, Vender JS, Fleming $A$. Clinical evaluation of continuous noninvasive blood pressure monitoring: accuracy and tracking capabilities. J Clin Monit 1995; 11 : 245-52.
3 Pace NL, East TD. Simultaneous comparison of intraarterial, oscillometric, and finapres monitoring during anesthesia. Anesth Analg 1991; 73: 213-20.

4 White WB, Lund-Johansen P, Omvik P. Assessment of four ambulatory blood pressure monitors and measurements by clinicians versus intraarterial blood pressure at rest and during exercise. Am J Cardiol 1990; 65: 60-6.

5 Graettinger WF, Lipson JL, Cheung DG, Weber MA. Validation of portable noninvasive blood pressure monitoring devices: comparisons with intra-arterial and sphygmomanometer measurements. Am Heart J 1988; 116: 1155-60.

6 Sapinski A. Comparison of the sphygmooscillographic method with the direct and auscultatory methods of measuring blood pressure. J Clin Monit 1994; 10: 373-6.

7 Gorback MS, Quill TJ, Graubert DA. The accuracy of rapid oscillometric blood pressure determination. Biomed Instrum Technol 1990; 24: 371-4.

8 N Kim-Gau, Small CF. Review of methods and simulators for evaluation of noninvasive blood pressure monitors. Journal of Clinical Engineering 1992; 17: 469-74.

9 Weinger MB, Scanlon TS, Miller L. A widely unappreciated cause of failure of an automatic noninvasive blood pressure monitor. J Clin Monit 1992; 8: 291-4.

10 Ghosh S. Statistical Design and Analysis of Industrial Experiments. New York: Marcel Dekker Inc., 1990: 253-4.

11 Christensen R. Mixed models and variance components. In: Plane Answers to Complex Questions; the Theory of Linear Models. New York: Springer-Verlag Inc., 1987: 223-40.

12 Quill TJ. Blood pressure monitoring. In: Anesthesia Equipment: Principles and Applications: St. Louis: Mosby-Year Book Inc., 1993: 274-83. 